R. J. Cohen and W. T. Sullivan, III, eds.

\title{
Educating the Public About Interference to Radio Observatories
}

\author{
David G. Finley ${ }^{1}$ \\ National Radio Astronomy Observatory, P.O. Box O, Socorro, New \\ Mexico 87801 USA
}

\begin{abstract}
Educating the public about interference to radio observatories is a different and more difficult task than educating the public about light pollution. Convincing and successful arguments against light pollution can be based on aesthetic, economic, cultural, safety and security considerations without relying solely on the need to preserve the environment for astronomy. In contrast, it is necessary to first convince members of the public of the value of radio astronomical research before making the case for interference protection. Once this is done, arguments about interference must be presented in ways understandable to a public that is, by and large, woefully uninformed about the technology involved. Successful approaches often borrow from the language of environmental protection and draw parallels to such issues as air and water pollution in justifying the expense of engineering measures to protect radio astronomy.
\end{abstract}

\section{Introduction}

Public support is a vital element in any effort to protect radio observatories from harmful interference that jeopardizes their ability to advance our knowledge of the Universe. While the support of the general public will not, by itself, ensure success in our efforts to protect the radio astronomical spectrum, lack of public support would certainly doom such efforts. The battle for spectrum protection is at its heart a political battle, and thus must be fought, at least in part, in the arena of public opinion.

This fact has been recognized by the optical-astronomy community in their efforts to reduce light pollution. The numerous successes at various governmental levels in implementing regulations to reduce light pollution have resulted from campaigns that convinced diverse segments of the population to support such measures.

While radio astronomers can follow such an example in building public support, they face significant obstacles not faced in the light-pollution battle. First, light pollution can be opposed on a number of grounds that resonate with people who have little or no interest in astronomy. The economic, aesthetic, cultural, safety and security benefits of reducing light pollution attract broad

\footnotetext{
${ }^{1}$ The National Radio Astronomy Observatory is a facility of the National Science Foundation, operated under cooperative agreement by Associated Universities, Inc.
} 
support without the need to convince people to protect astronomical research. On the other hand, measures to mitigate interference to radio observatories do not produce a similar set of non-astronomical benefits. In addition, radio interference is a highly technical issue that is beyond the understanding of much of the public, making the task of gaining public support more difficult.

Successful efforts to build public support for protecting the radio astronomical spectrum thus must be built on a foundation of public support for astronomy itself. The arguments for spectrum protection then must be made in non-technical terms understandable to the public.

\section{Public Involvement in Policy Issues}

The public faces a wide variety of policy issues, and most individuals devote the time to become informed or active about only a small number of these. For any specific, specialized issue, the level of public interest and involvement has been described as a pyramidal structure (Almond 1950). In this pyramid (Figure 1 ), officials with the power to enact policy measures sit at the pinnacle. Below them is a group of experts who actively lead public opinion on the issue. Among the general public, those who are interested in the issue are divided among the attentive public and the interested public. The attentive public is generally more active in support of their viewpoint on the issue, while members of the interested public are not active. At the bottom of the pyramid, with the largest numbers, are members of the residual public, who have little or no interest in the issue.

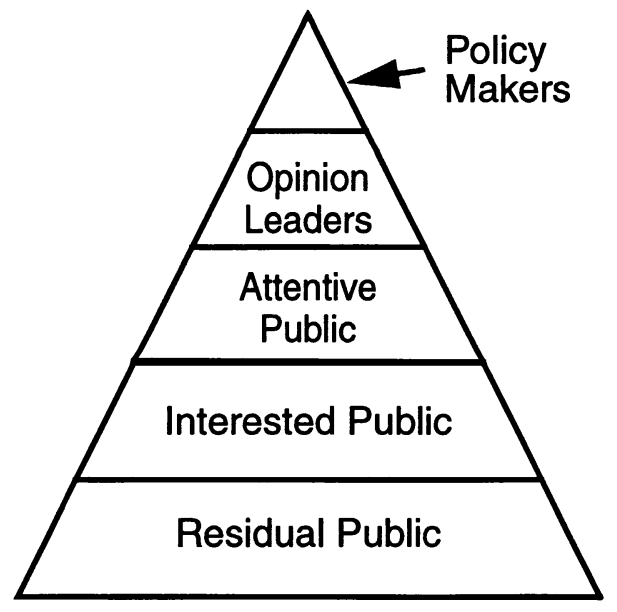

Figure 1. Public Involvement in Policy Issues.

In the United States, only 14 percent of adults are included in the attentive public for all science and technology policy issues (National Science Board 1998). Much of the success of the optical-astronomy community in gaining 
support for measures to control light pollution comes from the fact that their arguments effectively motivate many people from the large residual public for science policy issues. A campaign against light pollution can, in effect, build its own policy pyramid that includes many people with no interest in astronomical research. Unfortunately, that is not the case with the issue of interference to radio observatories.

Radio astronomers must assume the role of opinion leaders and work to educate the public about the interference issue. Our objective must be to increase the numbers of our attentive and interested publics for this issue, and to move members of the interested public up into the ranks of the attentive public, and thus of active supporters. Since the principal difference between an active member of the attentive public and a passive member of the interested public is a self-perception of being adequately informed on the issue (Miller 1999), our educational efforts should be directed toward giving members of the public confidence that they understand the interference problem and also our suggested remedies.

\section{The Value of Astronomy and of Radio Observations}

Astronomy has an excellent record of contributing to human progress from ancient times to the present. It has inherent cultural value in providing humans with a sense of their place in the Universe. It has produced numerous practical advances, and will continue to do so in the future. It has proved to be a powerful tool for science education and attracting young people to technical careers.

The inherent appeal of the night sky dates to prehistory and is part of cultures worldwide. Today, the basics of astronomical knowledge provide a conceptual framework for the universal human desire to understand our place in space and time.

The practical contributions of astronomy range from the calendar that helped early humans to progress beyond hunting and gathering to an agriculturebased society, to advances in modern medical imaging. The world-wide commerce we take for granted began because astronomers made it possible for mariners to navigate accurately across the oceans. Today, the communications and weather satellites upon which we depend represent a technology made possible by Kepler, Newton and the celestial mechanicians who followed them. Mathematics, computer science, telecommunications and other fields owe large debts to astronomy.

The visual appeal of astronomy and the excitement of new discoveries about the Universe make astronomy a powerful educational tool. People of all ages are drawn to science by the latest news from the frontiers of astrophysics. Astronomy serves as an entry point for a wide variety of scientific and technical careers, as young people first drawn to astronomy branch out into other technical fields that power today's high-tech economies. Non-scientists who maintain their interest in astronomy increase their scientific literacy, becoming more valuable as citizens, leaders and managers. Professional astronomers are aided greatly in their educational efforts by hundreds of thousands of amateur astronomers who volunteer their time to educate the public. 
The Universe provides us with a "cosmic laboratory" of extreme conditions that cannot be replicated on Earth. This laboratory represents an invaluable resource for all humanity. From it, we continue to gain fundamental new knowledge about physics - knowledge that in the future may spawn entirely new technologies.
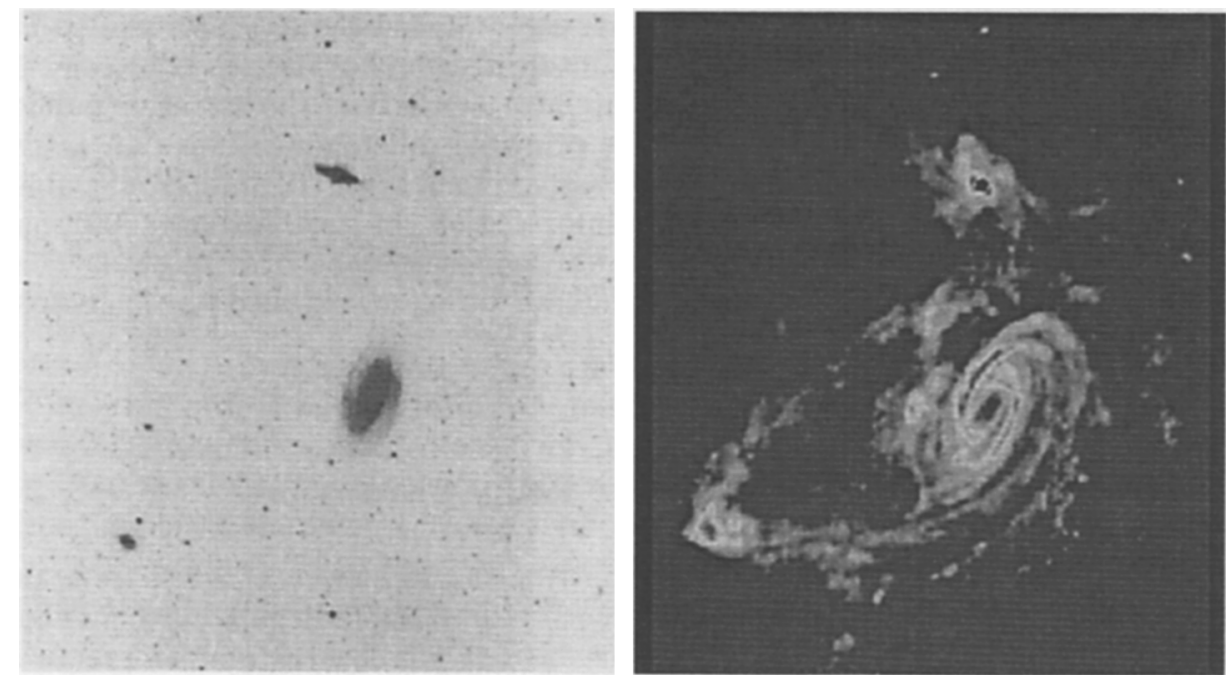

Figure 2. Radio astronomy helps show the "whole picture". Optical image (left) of the M81 Group of galaxies fails to show the interactions revealed by a VLA image at $21 \mathrm{~cm}$ wavelength (right) (Yun et al. 1994).

Radio astronomy, in a mere six decades of existence, has revolutionized our understanding of the Universe. From revealing the remnant radiation of the Big Bang to showing the afterglows of Gamma Ray Bursters, radio observers have provided unique insights. Six of the ten Nobel laureates cited for work in astronomy did that work with radio telescopes. For a wide variety of astronomical phenomena, radio observations provide essential pieces of the "whole picture" required for full understanding (Figure 2). Astronomy has become a multi-wavelength enterprise, and radio observations are an integral part of this effort.

Convincing people of the value astronomy - and radio observations - provide to society is the essential first step in educating the public about interference to radio astronomy. We have a good case and we must make it vigorously. Every astronomer should become familiar with the contributions our science has made and its promise for the future. In public lectures, classrooms, and in casual conversations, we must not be shy or defensive about the value of astronomy. Indeed, we must convey this message at every opportunity. Doing so will not only help in the fight to preserve the radio astronomical spectrum but, in most countries, will also help maintain support for public financing of astronomical institutions and efforts. 


\section{Obstacles to Public Education}

In the pyramidal structure of public involvement, our attentive public for the radio interference issue is very small, because few non-astronomers are aware of the problem or sufficiently knowledgeable about its technical aspects. Our interested public could include a majority of amateur astronomers and others who follow astronomical discoveries through the news media. Our rather formidable challenge is to move people upwards in the pyramid by gaining their attention for this issue and making them feel well informed about it.

The principal obstacles to effective public education are the public's lack of understanding of the technical issues surrounding interference mitigation and our own tendency to lapse into jargon when discussing the issue.

In an objective study of public knowledge, only 15 percent of U.S. adults were categorized as "civic scientificially literate", meaning that they understood a basic scientific vocabulary, understood the process of scientific research, and understood the impact of science on society (Miller 1999). Less than half of U.S. adults correctly answered that the Earth goes around the Sun once a year (National Science Board 1998).

Widespread anecdotal evidence suggests that many people fail to realize that "wireless" technologies such as mobile telephones, pagers, etc., rely on radio transmissions that could affect astronomy. In a survey of tourists at the Very Large Array (VLA) Visitor Center in New Mexico, 73 percent rated their own astronomical knowledge as "basic" or better. However, only 11 percent rated their radio astronomy knowledge above "basic", while 55 percent claimed no knowledge whatever of radio astronomy. These were tourists who had chosen to visit a radio observatory in a remote location. A majority of these tourists held college degrees and 78 percent had some college training.

A disturbingly large number of people on guided tours of the VLA are unaware of the difference between electromagnetic radiation and sound, and in fact seem quite surprised when disabused of the common misconception that radio astronomers "listen to sounds".

Clearly we are challenged to present information about radio astronomy and the interference issue in clear and accurate, but non-technical, terms understandable to a public largely unaware of the basics of our science. This requires serious effort to keep the language understandable and avoid lapsing into technical jargon. Jargon will intimidate our audience and raise their resistance to our message.

\section{Successful Approaches}

\subsection{Strategy}

First, we must take every opportunity to educate the public about radio astronomy and its contributions, through the news media, museums and science centers, observatory visitor centers, public lectures and other outlets. We need to do a better job of publicizing the results of radio astronomical research. While we rarely can compete with the dramatic visual impact of images from optical telescopes, radio astronomers continuously produce exciting new knowledge 
that, conveyed properly, captures the attention of the public and reinforces their support.

The importance of introductory college-level astronomy courses aimed at non-science majors cannot be overemphasized. The U.S. National Science Board's surveys have shown that those who have taken college science courses are much more likely to retain an interest in science policy issues and to support science. Thus, it is vital that radio astronomy and its contributions be well represented in astronomy courses aimed at non-science majors. This can be achieved by having radio astronomers teach such courses and by working with textbook and educational media publishers to assure that the work of radio observatories is included in the curriculum for these courses.

In specifically addressing the radio-interference problem, we need to define the issue in our own terms - terms that are both understandable to non-scientists and favorable to our cause. By thus defining the issue, we gain the high ground in the debate. Here we can follow the example of the optical-astronomy community and borrow from the language of environmental protection.
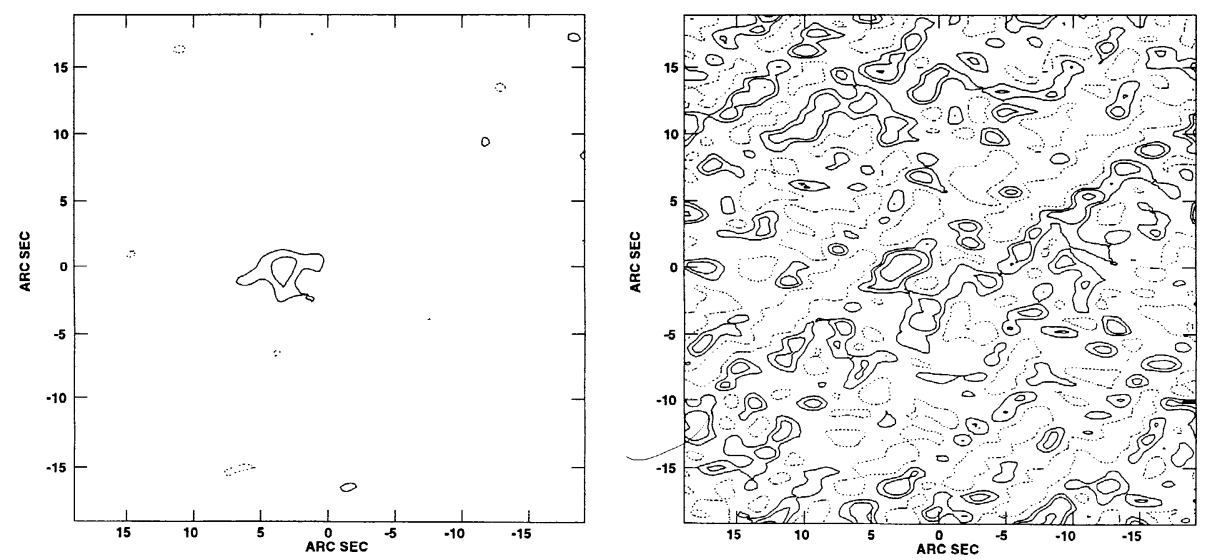

Figure 3. The effect of interference on radio images. A VLA image of an $\mathrm{OH} / \mathrm{IR}$ star observed at $1612 \mathrm{MHz}$ with no satellite signal present (left) and with an Iridium satellite approximately 22 degrees from the star (right). The increased noise in the image with the satellite present is clearly obvious, even to a non-technical audience. Courtesy G.B. Taylor, NRAO/AUI.

Our campaign against radio interference should use phrases such as "polluting" interference, "radio waste", "spillover" and "dirty" transmitters. Harmonics and spurious emissions should be portrayed as trespassers and polluters into territory where they do not belong. Simple illustrations of spillover into radio-astronomy bands and of the effect of interference on radio images (Figure $3)$ can effectively convey our message.

As reported elsewhere in this volume, radio astronomy may not remain the lone victim of interference problems. We were the first because of the extreme sensitivity of our systems, but other users of the radio-frequency spectrum are 
becoming vulnerable. This "canary in the mine" status of radio astronomy can strike a responsive chord in many minds.

In pointing out the value of astronomical research within the unique "cosmic laboratory", we must point out that our use of this invaluable resource can be degraded or prevented by radio interference. Those who indiscriminately pollute the radio spectrum are thus cutting off the rest of humankind from an important source of scientific knowledge and technological progress. No one, we must argue, has the right to do so.

We are, of course, in favour of efficient use of the radio spectrum, but only in a responsible manner that also allows astronomical research to continue. This position is similar to the successful arguments that convinced public policy makers that industry should not only make profits but also protect the air, water and soil from pollution. Counterarguments that measures to mitigate pollution would cost too much have largely been unsuccessful. In the U.S., public-opinion polls for the past decade have shown strong support (by a margin of more than 2-1) for protecting the environment even at the risk of curbing the economy (Gillespie 1999). We should tap this strong source of public support.

\subsection{Tactics}

When addressing a specific audience on this issue, one must carefully gauge the appropriate technical level of the discussion to avoid intimidating the listeners. It is better to keep the discussion simple, and keep the audience confident in their understanding, than to try covering all technical aspects and turn off the listeners. Questions from a more sophisticated member of the audience can be answered at the level of the question, but then the discussion should be returned to a level appropriate to the majority of the audience.

In keeping the public discussion simple, we once again can follow the successful example of campaigns for environmental protection. Preventing and remediating air, water and soil pollution are, in fact, complex and sophisticated technical disciplines. However, public discussion about protecting these resources rarely reaches the level of complexity facing professionals in those fields. The public simply wants the resources protected and most people feel little need to learn the details of how that is done.

In dealing with news media, it is important to recognize the differences among various types of media. In a television or radio news show, two or three minutes of air time would constitute significant coverage for that show, but would mean we must make our case very quickly in accurate but simple language. In discussing the issue with reporters for print media, we can be more complete and, depending on the publication and the reporter's own technical background, delve into some of the more technical aspects. Because of the great differences in the level of discussion possible in different media, separate press releases, tailored to these differences, may be advisable.

The more informed members of the public tend to get more of their information from newspapers and magazines than from broadcast outlets, so, at least initially, coverage in these media may prove more productive.

Finally, we must not overlook the growing importance of the World Wide Web as a means of information distribution, particularly to the more technicallysophisticated public. Radio observatories and other astronomical institutions 
should provide information on the interference issue aimed at an interested, but non-technical, public audience in their Web sites.

\section{Summary}

Radio astronomers must take an active role as leaders of public opinion to build broad support for protecting radio observatories from interference. The starting point for this effort is to aggressively promote the value and contributions to society as a whole of astronomy in general and radio observations in particular. We must all familiarize ourselves with astronomy's solid record of contributing to human progress and the promise for continuing to do so in the future. Then, we must take every opportunity to make this case to our fellow citizens.

In addressing the specifics of interference and its effects on radio observations, we must recognize that we are dealing with a public that is not welleducated technically and that will not respond well to intricate, jargon-laced technical discussions. Instead, we can convince a generally receptive public by using the language of protecting from pollution a valuable resource for all humanity. By defining the issue in these terms and placing the burden on those who for economic gain would deprive all humans of the benefits of knowledge to be gained from the "cosmic laboratory", we can build a troad base of public support.

\section{References}

Almond, G. A. 1950, The American People and Foreign Policy, New York: Harcourt, Brace

Gillespie, M. 1999, U.S. Public Worries About Toxic Waste, Air and Water Pollution as Key Environmental Threats, Princeton, N.J.: The Gallup Organization

Miller, J. D. 1999, "Scientific Literacy, Issue Attentiveness, and Attitudes toward Science and Space Exploration", paper presented to AAS meeting, May 1999

National Science Board 1998, Science $\mathcal{E}$ Engineering Indicators, Washington: U.S. Government Printing Office

Yun, M.S., Ho, P.T.P. and Lo, K.Y. 1994, Nature, 372, 530 